\title{
Electron beam cooling in intense focussed laser pulses
}

\author{
Samuel R. Yoffe, Adam Noble, Alexander J. Macleod, and Dino A. Jaroszynski \\ Department of Physics, SUPA, University of Strathclyde, Glasgow G4 0NG, UK
}

\begin{abstract}
In the coming years, a new generation of high-power laser facilities (such as the Extreme Light Infrastructure) will become operational, for which it is important to understand how the interaction with intense laser pulses affects the bulk properties of relativistic electron bunches. At such high field intensities, we expect both radiation reaction and quantum effects to have a dominant role to play in determining the dynamics. The reduction in relative energy spread (beam cooling) at the expense of mean beam energy predicted by classical theories of radiation reaction has been shown to occur equally in the longitudinal and transverse directions, whereas this symmetry is broken when the theory is extended to approximate certain quantum effects. The reduction in longitudinal cooling suggests that the effects of radiation reaction could be better observed in measurements of the transverse distribution, which for real-world laser pulses motivates the investigation of the angular dependence of the interaction. Using a stochastic single-photon emission model with a (Gaussian beam) focussed pulse, we find strong angular dependence of the stochastic heating.
\end{abstract}

Keywords: Radiation reaction, quantum effects, semi-classical model, beam cooling, stochastic photon emission, interaction angle, focussed laser pulses.

\section{INTRODUCTION}

The next few years will see next-generation high-power laser facilities (such as the Extreme Light Infrastructure $(\mathrm{ELI})^{1}$ ) become operational, delivering laser intensities well beyond those currently available. The extremely large fields generated by these pulses will allow new physical regimes, currently merely a playground for theorists, to be explored experimentally for the first time. In these regimes, it is expected that radiation reaction and quantum effects will not just be important, but will in fact dominate the dynamics.

It is well established that the motion of a moving charged particle can be described by the Lorentz force for a particle of mass $m$ and charge $q$ in an electromagnetic field $F$,

$$
\ddot{x}^{a}=\frac{q}{m} F^{a b} \dot{x}_{b} \quad \text { or } \quad \frac{d \vec{p}}{d t}=q\left(\vec{E}+\frac{\vec{p}}{\gamma m} \times \vec{B}\right),
$$

where a dot represents differentiation with respect to proper time, $s$. However, an accelerating charge radiates energy (and momentum), and as it does it recoils in order to conserve energy and momentum, altering the particle's trajectory. This is the concept of radiation reaction and it is unaccounted for in the Lorentz equation above. In many cases, this effect is extremely small and can be safely neglected, however as we move to higher field stengths this is no longer the case.

Despite more than a century of investigation, radiation reaction remains a contentious area of physics. Perhaps the most rigorous classical description of radiation reaction for a point particle is that by Dirac, ${ }^{2}$ which was based on earlier (independent) attempts to include the effects of radiation emission by Lorentz ${ }^{3}$ and Abraham. ${ }^{4}$ Unusually, the resulting Lorentz-Abraham-Dirac (LAD) equation contains a third derivative (with respect to time) which permits so-called runaway solutions. These pathological solutions, for which a particle accelerates indefinitely without the action of a force, are unphysical and can be prevented only at the expense of violating

Further author information:

S.R.Y.: E-mail: sam.yoffe@strath.ac.uk

A.N.: E-mail: adam.noble@strath.ac.uk

A.J.M.: E-mail: alexander.macleod@strath.ac.uk

D.A.J.: E-mail: d.a.jaroszynski@strath.ac.uk 
causality. In this case, the particle may experience pre-acceleration and respond to a future force which has not yet acted on the particle. The recent review ${ }^{5}$ describes these problems and proposed solutions in greater detail.

To avoid the pathologies found with the LAD equation, the most widely-used classical description was introduced by Landau and Lifshitz. ${ }^{6}$ The radiation reaction (self-)force is treated as a small perturbation about the Lorentz force, ${ }^{7}$ and terms are collected up to leading-order in the characteristic time, $\tau:=q^{2} / 6 \pi m$, to give what is known as the Landau-Lifshitz equation:

$$
\ddot{x}^{a}=-\frac{q}{m} F^{a b} \dot{x}_{b}-\tau \frac{q}{m}\left(\partial_{c} F^{a b} \dot{x}_{b} \dot{x}^{c}-\frac{q}{m} \Delta^{a}{ }_{b} F^{b c} F_{c d} \dot{x}^{d}\right),
$$

where the $\dot{x}$-orthogonal projection $\Delta^{a}{ }_{b}:=\delta_{b}^{a}+\dot{x}^{a} \dot{x}_{b}$ ensures that $p^{a} p_{a}=-m^{2}$ (where $p^{a}=(\gamma m, \vec{p})$ ) is preserved so that the particle remains on shell. This classical equation has found significant use and there is mounting evidence to suggest that the theory is valid provided that quantum effects may be ignored. ${ }^{8,9}$ However, this will certainly not be the case at the extreme conditions expected at ELI, and in order to connect with experiment design we must attempt to describe some of these effects.

Quantum effects are typically considered to be negligible if the electric field observed by the particle $\hat{E}=\gamma E$ is much less than the Sauter-Schwinger critical field ${ }^{10,11}$ typical of QED processes, $E_{S}=1.32 \times 10^{18} \mathrm{~V} / \mathrm{m}$. In this case, the quantum nonlinearity parameter is small,

$$
\chi:=\frac{\hat{E}}{E_{S}}=\frac{e \hbar}{m_{e}^{2}} \sqrt{F^{a b} F_{a c} \dot{x}_{b} \dot{x}^{c}} \ll 1,
$$

where $\ddot{x}_{0}^{a}=F^{a b} \dot{x}_{b}$ is the acceleration due to the Lorentz force only. ELI is expected to approach $\chi \sim 0.8$, where quantum effects will play an important part.

One issue with using the classical model when $\chi$ becomes large is that the theory considers radiation at all frequencies. Classically, there is nothing to prevent a particle radiating a small amount of energy at a high frequency, whereas in the quantum world an entire photon has to be emitted. This limits the available emission spectrum, since the particle can never radiate more energy than it actually has, such that classical theories overestimate radiation emission as quantum effects become important. ${ }^{12}$ Provided parameters are used such that $\chi^{2} \ll 1$ and the system is in some sense weakly quantum, then a semi-classical modification to (2) could be made ${ }^{13}$ and the emission reduced by scaling the radiation reaction force with a decreasing function of the quantum nonlinearity parameter, ${ }^{14} g(\chi)$. The full expression involves a non-trivial integral over Bessel functions, so instead we use the approximation introduced by Thomas et al. ${ }^{15 *}$

$$
g(\chi)=\left(1+12 \chi+31 \chi^{2}+3.7 \chi^{3}\right)^{-4 / 9}
$$

Predictions using this semi-classical model can be compared to those obtained from the classical Landau-Lifshitz theory in order to explore the role of quantum effects in the weakly quantum regime which will be available at ELI.

In this paper, we focus on the reduction of beam cooling predicted by the semi-classical model ${ }^{18}$ and the observation of a loss of symmetry, which motivates investigation of the interaction angle of a bunch colliding with a focussed laser pulse.

\section{PARTICLE DISTRIBUTION FUNCTION AND PHASE SPACE CONTRACTION}

The evolution of a particle distribution $\mathscr{F}(x, u)$ can be described by the Vlasov equation,

$$
\frac{d}{d s}(\mathscr{F} \mathcal{V})=\left[\frac{d \mathscr{F}}{d s}+\beta_{s} \mathscr{F}\right] \mathcal{V}=0
$$

where $\mathcal{V}$ is the phase-space volume element, $\beta_{s}(x, u)$ describes the rate of change of $\mathcal{V}$ with proper time, and $u^{a}=(\gamma, \vec{u})$ is the 4-velocity. We introduce the (null) wavevector $k^{a}=(\omega, \vec{k})$ and work with the phase $\eta=$

\footnotetext{
${ }^{*}$ Note that other approximations are available, such as ${ }^{16,17} g(\chi)=\left(1+18 \chi+69 \chi^{2}+73 \chi^{3}+5.806 \chi^{4}\right)^{-1 / 3}$.
} 
$-k \cdot x=\omega t-\vec{k} \cdot \vec{x}$ and Cartesian spatial coordinates $(\xi, \sigma, \zeta)$, along with the corresponding velocity components $u_{\eta}, u_{\xi}$ and $u_{\sigma}$. The final component is not independent, and can be found from the normalisation condition $u^{a} u_{a}=\left(u_{\eta} / \omega\right)\left(2 u_{\zeta}-u_{\eta} / \omega\right)+u_{\xi}^{2}+u_{\sigma}^{2}=-1$.

For a plane wave with arbitrary polarisation, the electromagnetic field tensor $F$ depends on spacetime only through the phase $\eta$ and can be written

$$
\frac{q}{m} F_{b}^{a}=\mathcal{E}_{\epsilon}(\eta)\left(\epsilon^{a} k_{b}-k^{a} \epsilon_{b}\right)+\mathcal{E}_{\lambda}(\eta)\left(\lambda^{a} k_{b}-k^{a} \lambda_{b}\right),
$$

where $\epsilon, \lambda$ are the (transverse) polarisation vectors and the functions $\mathcal{E}_{\epsilon, \lambda}(\eta)$ are dimensionless measures of the electric field strength. Similarly, we assume that the particle distribution also depends on spacetime only through the phase, such that $\mathscr{F}=\mathscr{F}\left(\eta, u_{\eta}, u_{\xi}, u_{\sigma}\right)$ and the Vlasov equation becomes

$$
u_{\eta} \frac{\partial \mathscr{F}}{\partial \eta}+\mathcal{A}^{I} \frac{\partial \mathscr{F}}{\partial u^{I}}+u_{\eta} \frac{\partial}{\partial u^{I}}\left(\frac{\mathcal{A}^{I}}{u_{\eta}}\right) \mathscr{F}=0
$$

where $u^{I} \in\left\{u_{\eta}, u_{\xi}, u_{\sigma}\right\}$ and the accelerations $\mathcal{A}^{I} \in\left\{\mathcal{A}_{\eta}, \mathcal{A}_{\xi}, \mathcal{A}_{\sigma}\right\}$ follow from the single-particle equations of motion. After division by $u_{\eta}>0$, the equation is expressed

$$
\frac{d \mathscr{F}}{d \eta}+\beta \mathscr{F}=0, \quad \text { where } \quad \beta=\frac{\partial}{\partial u^{I}}\left(\frac{\mathcal{A}^{I}}{u_{\eta}}\right) .
$$

It is the quantity $\beta$ which is responsible for any expansion $(\beta>0$, heating) or contraction $(\beta<0$, cooling) of the distribution (and the associated change in entropy ${ }^{19}$ ).

For the interaction of a highly relativistic particle bunch with an intense laser pulse, it is primarily the dependence of $\mathscr{F}$ on $\eta$ and $u_{\eta}$ which is of interest. We therefore integrate out the transverse degrees of freedom ${ }^{18}$ and define the longitudinal distribution,

$$
f\left(\eta, u_{\eta}\right)=\int_{\mathbb{R}^{2}} \mathscr{F} d u_{\xi} d u_{\sigma},
$$

which satisfies the reduced Vlasov equation

$$
\frac{d f}{d \eta}+\beta_{\|} f=0, \quad \text { where } \quad \beta_{\|}=\frac{\partial}{\partial u_{\eta}}\left(\frac{\mathcal{A}_{\eta}}{u_{\eta}}\right) .
$$

The quantity $\beta_{\|}$describes the longitudinal phase space contraction, such that the transverse may be found as

$$
\beta_{\perp}=\beta-\beta_{\|}=\frac{\partial}{\partial u_{\xi}}\left(\frac{A_{\xi}}{u_{\eta}}\right)+\frac{\partial}{\partial u_{\sigma}}\left(\frac{A_{\sigma}}{u_{\eta}}\right) .
$$

To gain any further information, we must make a choice of single-particle equations of the motion to use. We begin by considering the Landau-Lifshitz equations (indicated by $\hat{\mathcal{A}}$ ) which in our coordinates may be written:

$$
\begin{aligned}
& \hat{\mathcal{A}}_{\eta}=-\tau \underline{\mathcal{E}}^{2} u_{\eta}^{3} \\
& \hat{\mathcal{A}}_{\xi}=-u_{\eta}\left(\mathcal{E}_{\epsilon}+\tau u_{\eta} \mathcal{E}_{\epsilon}^{\prime}\right)-\tau \underline{\mathcal{E}}^{2} u_{\eta}^{2} u_{\xi} \\
& \hat{\mathcal{A}}_{\sigma}=-u_{\eta}\left(\mathcal{E}_{\lambda}+\tau u_{\eta} \mathcal{E}_{\lambda}^{\prime}\right)-\tau \underline{\mathcal{E}}^{2} u_{\eta}^{2} u_{\sigma},
\end{aligned}
$$

where $\underline{\mathcal{E}}^{2}=\mathcal{E}_{\epsilon}^{2}+\mathcal{E}_{\lambda}^{2}$ and prime denotes differentiation with respect to $\eta$. For the classical Landau-Lifshitz theory we therefore find

$$
\hat{\beta}_{\|}=\hat{\beta}_{\perp}=-2 \tau \underline{\mathcal{E}}^{2} u_{\eta} \leq 0 .
$$

Since this is negative (semi-definite), any change to the distribution as the particles evolve must correspond to a cooling - a reduction in the relative energy/momentum spread of the particles. Moreover, we see that equal cooling occurs in the longitudinal and transverse directions. 

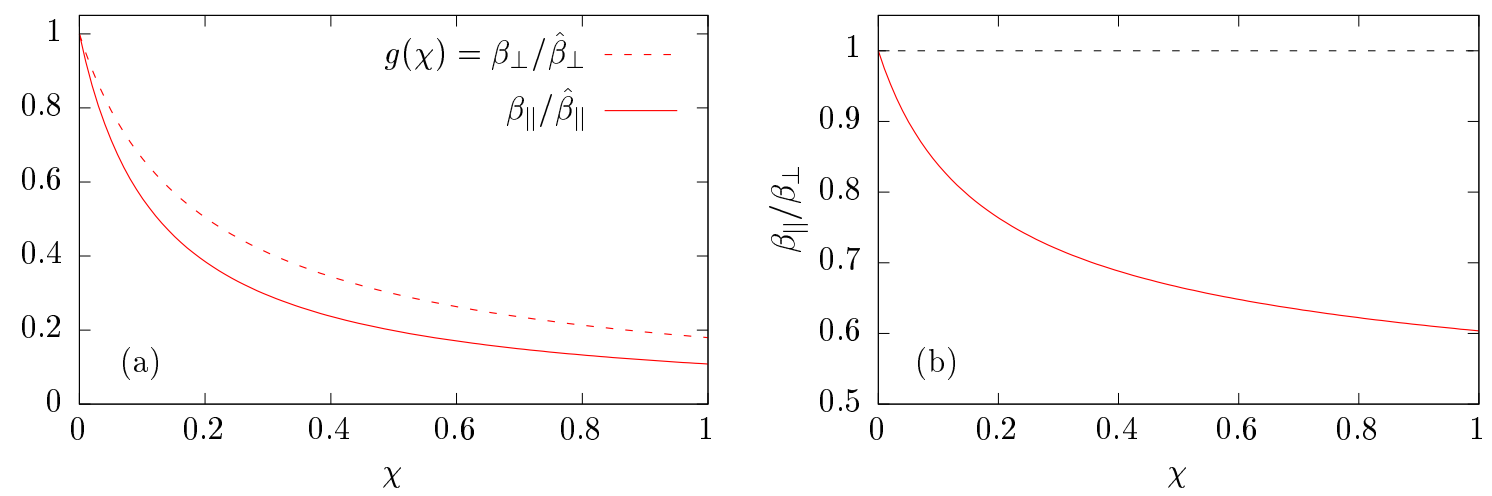

Figure 1. (Colour online.) Part (a): transverse and longitudinal beam cooling for the semi-classical model compared to that for the classical Landau-Lifshitz prediction. Part (b): comparison of longitudinal and transverse beam cooling for the semi-classical model as the system becomes more quantum.

Let us now extend the classical theory for use in the weakly quantum regime and consider the equations of motion for the semi-classical model. This amounts to a rescaling $\tau \rightarrow \tau g(\chi)$ in equations (12), specifically

$$
\begin{aligned}
& \mathcal{A}_{\eta}=-\tau g(\chi) \underline{\mathcal{E}}^{2} u_{\eta}^{3} \\
& \mathcal{A}_{\xi}=-u_{\eta}\left(\mathcal{E}_{\epsilon}+\tau g(\chi) u_{\eta} \mathcal{E}_{\epsilon}^{\prime}\right)-\tau g(\chi) \underline{\mathcal{E}}^{2} u_{\eta}^{2} u_{\xi} \\
& \mathcal{A}_{\sigma}=-u_{\eta}\left(\mathcal{E}_{\lambda}+\tau g(\chi) u_{\eta} \mathcal{E}_{\lambda}^{\prime}\right)-\tau g(\chi) \underline{\mathcal{E}}^{2} u_{\eta}^{2} u_{\sigma} .
\end{aligned}
$$

Since in the plane wave we have $\chi=\chi\left(\eta, u_{\eta}\right)=3 \tau \underline{\mathcal{E}} u_{\eta} / 2 \alpha$ (where $\alpha$ is the fine structure constant) the transverse contraction $\beta_{\perp}$ is simply scaled by $g(\chi)$; whereas, the longitudinal component picks up an additional contribution due to the derivative of $g(\chi)$ :

$$
\beta_{\perp}=g \hat{\beta}_{\perp} \quad \text { and } \quad \beta_{\|}=g \hat{\beta}_{\|}+\frac{\partial g}{\partial u_{\eta}} \frac{\hat{\mathcal{A}}_{\eta}}{u_{\eta}} .
$$

Using the approximation for $g(\chi)$ given by (4), we find

$$
\frac{\partial g}{\partial u_{\eta}} \frac{\hat{\mathcal{A}}_{\eta}}{u_{\eta}}=-\beta_{\perp}\left[\frac{2}{9} \chi g^{9 / 4}(\chi)\left(12+62 \chi+11.1 \chi^{2}\right)\right] \geq 0,
$$

which corresponds to heating. Thus, as the system becomes more strongly quantum, there is a general reduction to beam cooling due to $g(\chi)<1$; but there is also an additional reduction to the longitudinal cooling which breaks the symmetry between the longitudinal and transverse contributions.

Figure 1 shows the relative variation of the longitudinal and transverse phase space contraction as quantum effects become more and more important. As $\chi$ increases, not only does the semi-classical model predict substantially less beam cooling than the Landau-Lifshitz theory (a) but the anisotropy of the cooling increases (b).

This reduction in longitudinal - by which we mean parallel to the laser propagation direction - beam cooling could be used to help inform experiments looking to measure the effects of radiation reaction. Varying the interaction angle of the incoming electron bunch and laser pulse, rather than having them meet head on, could help maximise any cooling signiture present in the final state. In order to investigate this, we must first address two limitations of the work so far: the plane wave approximation; and stochastic heating introduced when continuous classical emission is no longer applicable.

\section{FOCUSSED LASER PULSES AND STOCHASTIC HEATING}

While a useful tool for analytical studies, the plane wave approximation is rarely useful for comparison to experiment due to the strong focussing required to obtain high laser intensity. In addition, the concept of 

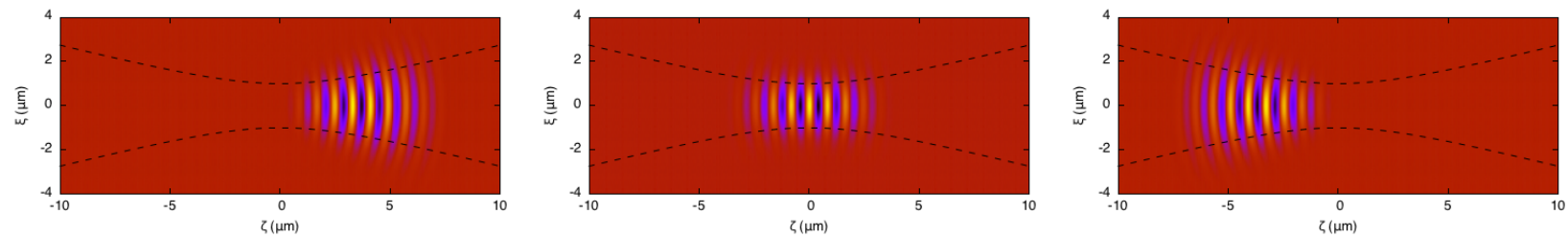

Figure 2. (Colour online.) Gaussian laser beam with waist $w_{0}=1 \mu \mathrm{m}$ (propagating in $-\zeta$ direction) before (left), during (centre) and after (right) the interaction with an electron bunch (moving in $+\zeta$ direction).

changing the interaction angle with a plane wave is not very intuitive. To overcome this, we instead consider the field tensor to have full spacetime dependence and write

$$
\frac{q}{m} F^{a b}=\left(\partial^{a} \Phi\right)\left(\mu^{b}+k^{b} / \omega\right)-\left(\partial^{b} \Phi\right)\left(\mu^{a}+k^{a} / \omega\right)+\left(\partial^{a} a_{\epsilon}\right) \epsilon^{b}-\left(\partial^{b} a_{\epsilon}\right) \epsilon^{a}+\left(\partial^{a} a_{\lambda}\right) \lambda^{b}-\left(\partial^{b} a_{\lambda}\right) \lambda^{a},
$$

where $\Phi$ is the scalar potential which is found using the Lorenz gauge condition (using a procedure similar to Harvey et al..$\left.^{20}\right) \quad \partial_{b} A^{b}=0$ with the vector potential $A^{b}=\left(\Phi, a_{\epsilon}, a_{\lambda}, 0\right)$. For the following, we consider the circularly-polarised Gaussian laser beam described by

$$
a_{\epsilon}-i a_{\lambda}=a_{0} \frac{w_{0}}{w(\zeta)} \sin ^{2}\left(\frac{\eta}{2 N}\right) \exp \left[-\frac{\xi^{2}+\sigma^{2}}{w^{2}(\zeta)}\right] \exp i\left[\eta+\left(\frac{\zeta-\zeta_{f}}{z_{R}}\right)\left(\frac{\xi^{2}+\sigma^{2}}{w^{2}(\zeta)}\right)-\tan ^{-1}\left(\frac{\zeta-\zeta_{f}}{z_{R}}\right)\right],
$$

where $a_{0}$ is a dimensionless measure of the peak laser intensity, $N$ is the number of cycles, $z_{R}=\pi w_{0}^{2} / \lambda_{0}$ is the Rayleigh length for a laser with wavelength $\lambda_{0}$, and the waist is given by $w(\zeta)=w_{0} \sqrt{1+\left[\left(\zeta-\zeta_{f}\right) / z_{R}\right]^{2}}$ with $w_{0}$ the radius at the position of the focus, $\zeta_{f}$. This is illustrated in Figure 2 for $\zeta_{f}=0$.

In addition to using a focussed laser pulse in place of the plane wave, we move to a stochastic model for single photon emission based on the probability of photon emission derived from QED. ${ }^{12,21,22}$ Starting from a large ensemble of initially-identical electrons (i.e. zero momentum spread), the random nature of the photon emission will cause the final properties to vary, depending on when and how much energy the electrons radiate. The final distribution is therefore expected to experience what is referred to as stochastic heating.

In order to look at the strength of the stochastic heating and its dependence on interaction angle, we consider an ensemble of 1000 relativistic electrons with initial Lorentz factor $\gamma_{0}=500$ colliding with a 10-cycle $(27$ fs) laser pulse with peak intensity $I=8.6 \times 10^{22} \mathrm{~W} / \mathrm{cm}^{2}$ and focussed waist $w_{0}=2.5 \mu \mathrm{m}$ at initial angle $\theta_{i} \in\left\{0^{\circ}, 30^{\circ}, 60^{\circ}, 90^{\circ}, 120^{\circ}, 150^{\circ}\right\}$. A selection of example trajectories are shown in Figure 3 for four different $\theta_{i}$. We see that, for $\theta_{i}=0^{\circ}$, it is common to have the electrons deflected into positive and negative angles, or even turned around completely, but as we move through to $60^{\circ}$ and beyond we favour increased angle.

The final properties of all of the electrons are then compiled to produce Figure 4. The deviation angle $\Delta \theta=\theta_{f}-\theta_{i}$ is plotted against final Lorentz factor $\gamma_{f}$ for each electron. Electrons that collide head on radiate most strongly, and emerge with an average $\bar{\gamma}=71$ substantially lower than they started with. There is also no preference to scatter into $\Delta \theta>0$ or $\Delta \theta<0$, although most end up with $-90^{\circ}<\Delta \theta<90^{\circ}$. We also see that, despite starting with zero energy spread, stochastic heating has caused a relative spread $\hat{\sigma}=\sigma / \bar{\gamma} \simeq 25 \%$ to develop.

As the initial angle is increased, the electrons tend to lose less energy and end up with larger $\bar{\gamma}$. As well as a clustering around smaller $\Delta \theta$, there is also a clear preference for scattering into $\Delta \theta>0$. This is due to the relative direction of laser propagation, as the electrons tend to be 'dragged' round by the laser. (Note that $\theta_{i}<0$ leads to $\Delta \theta<0$ dominating.)

Finally, it is interesting to note that the maximum stochastic heating does not occur for electrons colliding head on, despite the maximal loss of energy. Instead, maximal heating is observed for around $\theta_{i} \simeq 60^{\circ}$. 

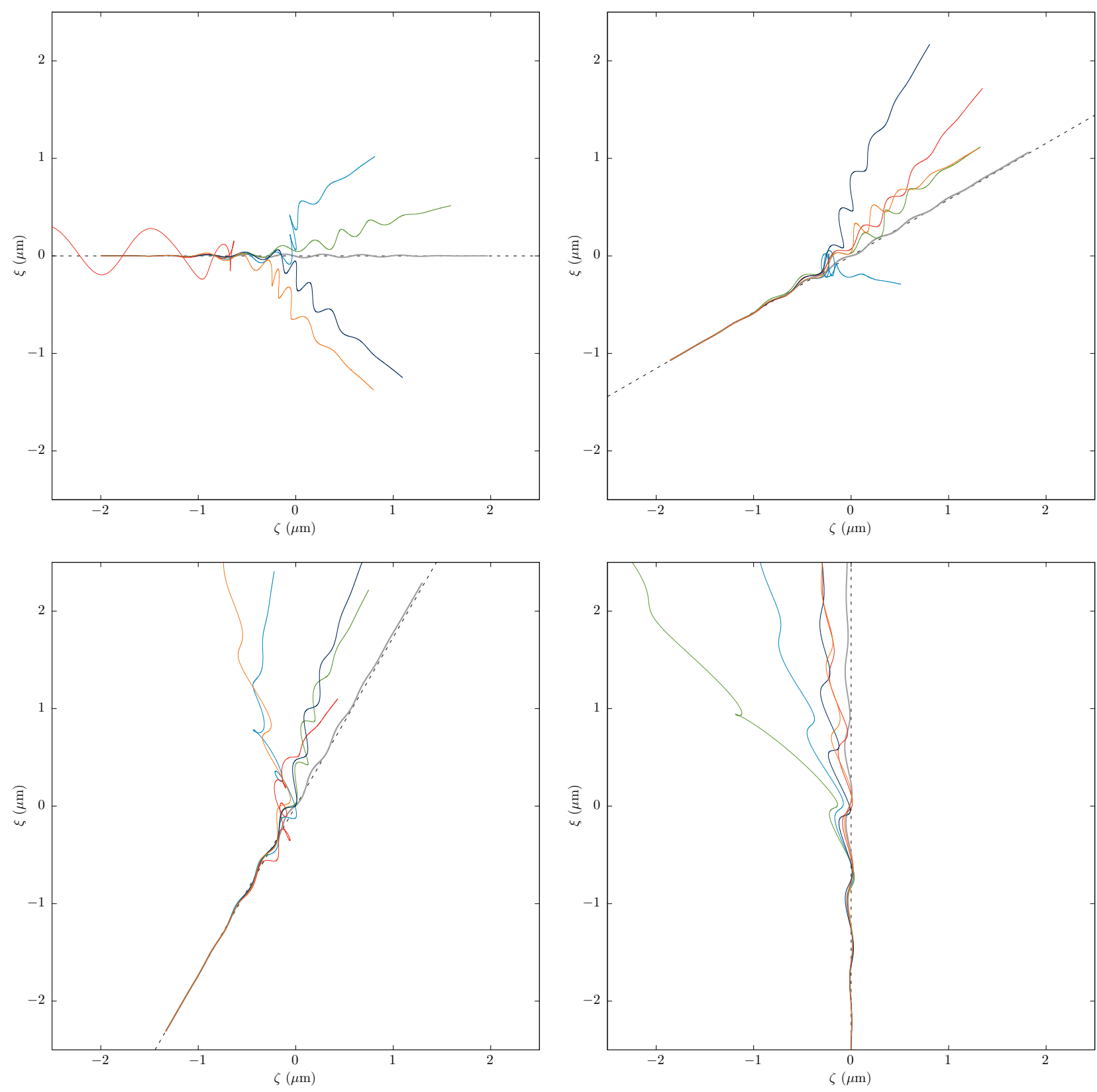

Figure 3. (Colour online.) Example trajectories for electrons with initial $\gamma_{0}=500$ colliding with a circularly-polarised laser with intensity $a_{0}=200\left(I=8.6 \times 10^{22} \mathrm{~W} / \mathrm{cm}^{2}\right)$ at initial angle (top left) $0^{\circ}$; (top right) $30^{\circ}$; (bottom left) $60^{\circ}$; and (bottom right) $90^{\circ}$. Dashed line indicates motion in a straight line, while grey shows the path of an electron follwing just the Lorentz force. 


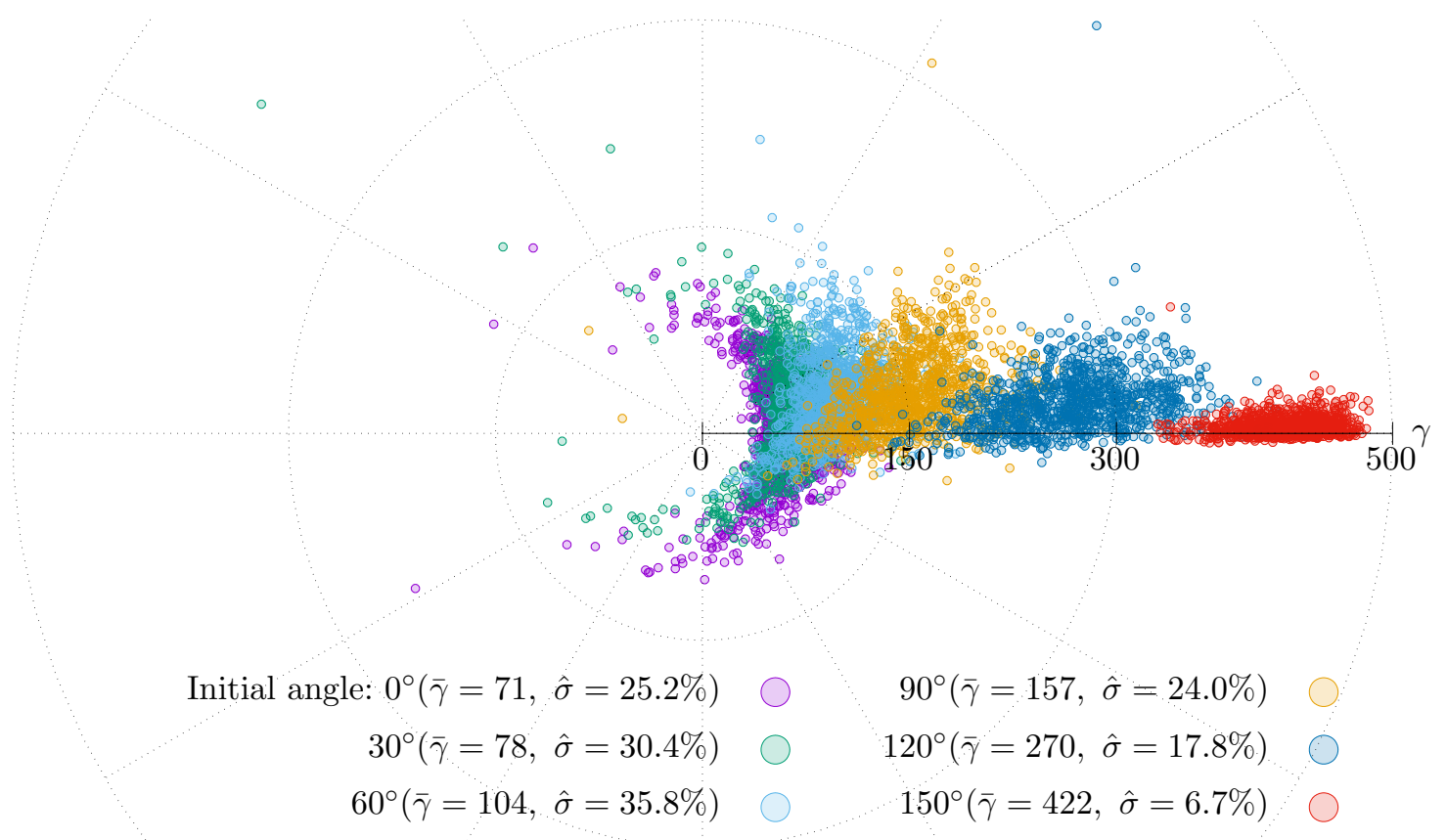

Figure 4. (Colour online.) Final electrons: Comparison of deviation angle $\Delta \theta=\theta_{f}-\theta_{i}$ and final energy $\gamma_{f}$.

\section{CONCLUSIONS}

With new, high-power laser facilities due to come online over the next few years, it is important to develop our understanding of the fundamental processes that underlie physics at unprecedented field strengths, and design experiments to measure effects that have until now been out of reach. At such facilities, not only will radiation reaction and quantum effects dominate the dynamics, requiring quantum models and rendering predictions made by classical theories unreliable, but it will also be increasingly important to move away from the plane wave approximation and instead turn our attention to more realistic finite pulses.

In this paper, we have demonstrated that the classical Landau-Lifshitz equations lead to a cooling of a relativistic electron beam when it collides with a high intensity laser pulse, and that this cooling is split equally between the longitudinal and transverse directions. However, in addition to reducing the overall beam cooling, the semi-classical model introduces a heating term to the longitudinal distribution which leads to an anisotropy in the cooling. This imbalance in the cooling of the longitudinal and transverse distributions suggests that experiments may find a particular interaction geometry beneficial for measuring maximum signal.

Using a stochastic single-photon emission model, the interaction of initially-identical electron ensembles colliding with a focussed pulse represented by a Gaussian beam demonstrated stochastic heating sensitive to the interaction angle, and a bias for final deviation (or scattering) angle. Whether this stochastic heating can in any way be compared to the heating contribution found for the semi-classical model is of interest for the application of efficient deterministic models when the system is no longer weakly quantum, and will be addressed in future work.

\section{ACKNOWLEDGMENTS}

This work is supported by the UK EPSRC (grants EP/J018171/1 and EP/N028694/1); the EC H2020 LaserlabEurope (grant 654148); EuCARD-2 (grant 312453); and EuPRAXIA (grant 653782). Results were obtained using the EPSRC-funded ARCHIE-WeSt High Performance Computer (www.archie-west.ac.uk; Grant EP/K000586/1). Dataset available online. ${ }^{23}$ 


\section{REFERENCES}

[1] http://www.eli-laser.eu/; http://www.eli-np.ro/.

[2] Dirac, P. A. M., "Classical Theory of Radiating Electrons," Proc. R. Soc. A 167, 148 (1938).

[3] Lorentz, H. A., [The Theory of Electrons and its Applications to the Phenomena of Light and Radiant Heat], Stechert, New York (1916).

[4] Abraham, M., [The Classical Theory of Electricity and Magnetism], Blackie, London (1932).

[5] Burton, D. A. and Noble, A., "Aspects of electromagnetic radiation reaction in strong fields," Contemporary Physics 55, 110-121 (2014).

[6] Landau, L. D. and Lifshitz, E. M., [The Classical Theory of Fields], Pergamon, London (1962).

[7] Yoffe, S. R., Noble, A., Kravets, Y., and Jaroszynski, D. A., "Cooling of relativistic electron beams in chirped laser pulses," Proc. SPIE 9509, 950905 (2015).

[8] Kravets, Y., Noble, A., and Jaroszynski, D. A., "Radiation reaction effects on the interaction of an electron with an intense laser pulse," Phys. Rev. E 88, 011201(R) (2013).

[9] Spohn, H., "The critical manifold of the Lorentz-Dirac equation," Europhys. Lett. 50, 287 (2000).

[10] Sauter, F., "Über das Verhalten eines Elektrons im homogenen elektrischen Feld nach der relativistischen Theorie Diracs," Zeitschrift fr Physik 82, 742 (1931).

[11] Schwinger, J., "On Gauge Invariance and Vacuum Polarization," Phys. Rev. 82, 664 (1951).

[12] Ritus, V. I., "Quantum effects of the interaction of elementary particles with an intense electromagnetic field," J. Sov. Laser Res. 6, 497-617 (1985).

[13] Erber, T., "High-energy electromagnetic conversion processes in intense magnetic fields," Rev. Mod. Phys. 38, 626 (1966).

[14] Kirk, J. G., Bell, A. R., and Arka, I., "Pair production in counter-propagating laser beams," Plasma Phys. Control. Fusion 51, 085008 (2009).

[15] Thomas, A. G. R., Ridgers, C. P., Bulanov, S. S., Griffin, B. J., and Mangles, S. P. D., "Strong RadiationDamping Effects in a Gamma-Ray Source Generated by the Interaction of a High-Intensity Laser with a Wakefield-Accelerated Electron Beam," Phys. Rev. X 2, 041004 (2012).

[16] Esirkepov, T. Z., Bulanov, S. S., Koga, J. K., Kando, M., Kondo, K., Rosanov, N. N., Korn, G., and Bulanov, S. V., "Attractors and chaos of electron dynamics in electromagnetic standing waves," Physics Letters A 379(36), 2044 - 2054 (2015).

[17] Kostyukov, I. Y. and Nerush, E. N., "Production and dynamics of positrons in ultrahigh intensity laser-foil interactions," Physics of Plasmas 23(9), 093119 (2016).

[18] Yoffe, S. R., Kravets, Y., Noble, A., and Jaroszynski, D. A., "Longitudinal and transverse cooling of relativistic electron beams in intense laser pulses," New Journal of Physics 17(5), 053025 (2015).

[19] Burton, D. A. and Noble, A., "On the entropy of radiation reaction," Phys. Lett. A 378, 1031 (2014).

[20] Harvey, C., Marklund, M., and Holkundkar, A. R., "Focusing effects in laser-electron thomson scattering," Phys. Rev. Accel. Beams 19, 094701 (Sep 2016).

[21] Green, D. G. and Harvey, C. N., "Transverse Spreading of Electrons in High-Intensity Laser Fields," Phys. Rev. Lett. 112, 164801 (2014).

[22] Yoffe, S., Noble, A., Macleod, A., and Jaroszynski, D., "Cooling of relativistic electron beams in intense laser pulses: Chirps and radiation," Nuclear Instruments and Methods in Physics Research Section A: Accelerators, Spectrometers, Detectors and Associated Equipment 829, 243 - 247 (2016).

[23] Yoffe, S. R., Noble, A., Macleod, A. J., and Jaroszynski, D. A., "Interaction angle dependence of stochastic photon emission in the collision of electrons with intense focussed laser pulses," (2017). http://dx.doi.org/10.15129/f56324dd-5f25-4775-86e1-087d09b3e75d. 